\title{
Der bundesstaatliche Landbesitz in den USA
}

Hans W. Zimmermann

\section{Zusammenfassung}

Die Bereitstellung von Neusiedelland war in den USA Bundessache, so daß der Staat zum größten Grundstückhändler aller Zeiten geworden ist. Mit fortschreitender Kolonisierung aber änderten sowohl die Ansichten über die Wünschbarkeit öffentlichen Landbesitzes als auch die Aufgaben, die den staatlichen Institutionen übertragen wurden. Der Wandel von altem zu neuem Arbeitsbereich des früheren "General Land Office» wird beschrieben.

\section{Einleitung}

Während über eines Jahrhunderts war die Landbeschaffung, die Bereitstellung von neuen Siedlungsgebieten, fast die Hauptforderung, welche von den Amerikanern an ihren jungen Staat gerichtet wurde. Der Erfüllung dieser Aufgabe diente ein beträchtlicher technischer Apparat, das "General Land Office». Fast unbekannt ist bei uns hingegen, wie diese erste Aufgabe durch neue, wichtigere ganz in den Hintergrund gedrängt worden ist. Andeutungsweise werden die kräftigen Wandlungen der alten Institution durch die Umtaufe des Verwaltungszweiges in "Bureau of Land Management» sichtbar. Die Wandlung dieses Amtes vom Händler zum Bewirtschafter soll hier skizziert werden. Die Angaben dazu stammen zum Teil aus Verwaltungsschriften, die in Europa kaum erhältlich sind, und von persönlichen Auskünften, die mir Herr Charles F. Switzer (Lakewood, Colorado), ein Funktionär dieser Institution, geben konnte. Dafür möchte ich ihm an dieser Stelle meinen besten Dank aussprechen.

\section{Die Entstehung des Staatslandes}

Beim Eindringen der Weißen in Nordamerika war nach europäischem Gewohnheitsrecht natürlich alles Land bereits in Besitz genommen, nämlich von den Indianern. Die ersten Siedler suchten daher, sich Land käuflich zu erwerben, d. h. im Tausch gegen Waren des täglichen Bedarfes. Dieses Verfahren mußte aber bei dem enormen Landhunger der Einwanderer bald seine natürlichen Grenzen finden, so daß der Landerwerb auf nicht mehr ganz rechtmäßigem Weg begann. Hierzu war der sonst gar nicht besonders geschätzte Staat das ideale Werkzeug, denn der einzelne Bürger fühlte sich nicht auf ihn verpflichtet, machte sich also die Hände nicht schmutzig, wenn die Regierung zu zweifelhaften Methoden griff: «Right or wrong, my country» war die ideale Maxime für die Situation. War einmal Areal gewonnen, so gehörte es naturgemäß dem Staat, von dem es der einzelne Bürger rechtmäßig erwerben konnte. Dadurch begannen auch die Staatseinnahmen zu fließen, während auf die bei den Siedlern von Europa her verhaßten direkten Steuern vorerst noch verzichtet werden konnte.

Die Idee, daß der Staat durch eine gezielte Landpolitik seine innern wirtschaftlichen und politischen Ziele zu erreichen sucht, ist alt. So beschaffte sich der alte römische Staat Ländereien (unter dem beschönigenden Ausdruck «redimere» - zurückkaufen), um einen Teil davon für die Entschädigung seiner Veteranen zu verwenden. Verschiedene der dreizehn alten Kolonien an der Ostküste Nordamerikas übernahmen den Gedanken fast vollständig gleich; mit ihren Veteranenkolonien war aber auch beabsichtigt, durch die bewährten Soldaten die Westgrenze gegen Indianer und Franzosen gesichert zu wissen ${ }^{8}$.

1783 geschah im Vertrag von Paris der rechtliche Übergang des Landes östlich des Mississippi von England an die USA. Nun hatten aber die dreizehn alten Kolonien schon lange eine Reihe von größern Gebietsansprüchen gegen Westen geltend gemacht, die sich teilweise kräftig überschnitten. Damit drohten der jungen Republik bereits ernste Zerwürfnisse. Die glückliche Überwindung der Krise wuchs aus der Tatsache heraus, daß damals weder Bundesstaat noch Einzelstaaten richtige soziale und gemeinwirtschaftliche Aufgaben erfüllten und auf keinem Zusammengehörigkeitsgefühl aufgebaut waren; niemand erstrebte flächenmäßig an Bedeutung gewinnende Staatswesen, sondern es war nur Neusiedelland gesucht. Deswegen fand der Vorschlag der Vertreter Marylands im Kongreß sofort eine Mehrheit, nämlich daß alle umstrittenen Gebiete dem neuen Bundesstaat als sogenannte Public Domain abgetreten würden 8 . Das größte Landstück wurde von Virginia eingegeben und später zu den Staaten West Virginia und Kentucky; North Carolinas Beitrag wurde zu Tennessee; Connecticut hatte den Nordosten von Ohio beansprucht und verzichtete jetzt. Pennsylvania und New York aber verstanden es, sich ihre Ansprüche im Westen zu sichern, so daß sie heute an die Großen Seen reichen 1 . 
Die juristische Grundlage der Public Domain war allerdings schon früher geschaffen worden, nämlich 1780 durch einen Kongreßbeschluß (zit. nach 3, S. 61): ... daß das noch nicht durch einen Siedler aufgenommene Land, welches von irgend einem der Einzelstaaten abgetreten oder überlassen wird ... zum gemeinsamen Nutzen der Vereinigten Staaten verwendet werden soll.» Aber zuerst mußten die Ansprüche Englands zurückgezogen werden, bevor richtig an die Arbeit gegangen werden konnte.

\section{Das Wachstum der Public Domain}

Die Ausdehnung des Staatslandes lief zu einem guten Teil, aber nicht vollständig, parallel zur territorialen Entwicklung der USA; in den spanischen Kolonialgebieten sind die bestehenden Landansprüche weißer Siedler zu großen Teilen gewahrt geblieben. In einer kleinen Chronologie soll das Wachstum kurz verfolgt werden ${ }^{8}$. Die Ziffern beziehen sich auf jene in der Karte 1:

\begin{tabular}{|c|c|c|}
\hline 1 & 1783 & Umstrittene Gebiete der dreize \\
\hline 2 & 1783 & $\begin{array}{l}\text { Vertrag von Paris: England trit } \\
\text { sippi ab (ohne Florida und Go }\end{array}$ \\
\hline 3 & 1803 & $\begin{array}{l}\text { Kauf Louisianas von Napole } \\
\text { Mississippi ohne Texas und zw }\end{array}$ \\
\hline $3 a$ & 1818 & $\begin{array}{l}\text { Nach Grenzbereinigung mit } \mathrm{K} \\
\text { nommen }\end{array}$ \\
\hline 4 & 1819 & Spanien tritt Florida und die ö \\
\hline 5 & 1845 & $\begin{array}{l}\text { Texas trennt sich von Mexikc } \\
\text { von den USA annektieren }\end{array}$ \\
\hline 6 & 1846 & $\begin{array}{l}\text { Oregon Compromise mit Eng } \\
\text { westens am } 48 \text {. Breitenkreis (C }\end{array}$ \\
\hline 7 & 1848 & $\begin{array}{l}\text { Abtretung von Kalifornien un } \\
\text { ten nach dem Krieg mit Mexil }\end{array}$ \\
\hline 8 & 1850 & Erwerb eines Restgebietes im \\
\hline 9 & 1853 & Gadsden-Kauf \\
\hline (10) & 1867 & Kauf Alaskas von Rußland fi \\
\hline (11) & 1898 & Annexion von Hawaii \\
\hline & & $\begin{array}{l}\text { Total Areal, das einmal zu } \\
\text { worden ist }\end{array}$ \\
\hline \multicolumn{3}{|c|}{$\begin{array}{l}\text { Diese riesige Fläche war natürlich nie auf einmal im } \\
\text { Besitz des Staates; so wie im Westen neues Land } \\
\text { beschafft wurde, ging es im Osten durch Verkauf } \\
\text { an neue Einwanderer weg, der Staat war Grund- } \\
\text { stückhändler. Die Mittel, deren er sich dazu be- } \\
\text { diente, waren, wie die Liste zeigt, vielfältig; das Aus- } \\
\text { maß der Entwicklung des Territoriums aber zeugt } \\
\text { von einem außerordentlichen Vertrauen in die Zu- } \\
\text { kunft. }\end{array}$} \\
\hline
\end{tabular}

\section{Die Abgabe von Land aus der Public Domain}

\section{Die Vorarbeiten}

Úber die neuerworbenen Gebiete gab es natürlich noch keine zuverlässigen Karten, erst recht nicht die nötigen Grundbuchpläne; die Siedler aber drängten. In der Law Ordinance von 1785 legte die Regierung daher das weitere Vorgehen fest, welches in drei Stufen erfolgen sollte:

1. Klärung (clearance) der indianischen Ansprüche

2. Vermessung des Landes

3. eigentlicher Verkauf
Millionen Acres Millionen km² 237 0,96

$304 \quad 1,23$

530

2,15

46

0,19

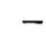

183

0,74

$339 \quad 1,37$

$76 \quad 0,31$

$19 \quad 0,07$

$375 \quad 1,52$
Zuerst wurden die indianischen Besitztitel geprüft, zum Teil anerkannt, zum Teil gelöscht (manchmal auch nachträglich); die Erledigung erfolgte aber recht summarisch, so daß auch heute noch Prozesse darum geführt werden müssen.

Für das zweite ernannte die Regierung Thomas Hutchinson zum «ersten Geographen der Nation.» Unter ihm entstand die quadratische Landvermessung, die sich an die Haupthimmelsrichtungen anlehnt.

Für das dritte wurde der juristisch einfachstmögliche Weg gesucht, damit das ganze Verfahren dem geistigen Niveau der «frontier» angepaßt sei ${ }^{8}$.

\section{Die technische Durchführung}

Die neue Vermessung umfaßte nicht alle Staaten; deren achtzehn im Osten, Teile von Ohio, ganz Texas und Hawaii gehörten nicht dazu. Die andern Gebiete erhielten eine «base line» und einen Hauptmeridian. Aus praktischen Gründen waren es 43 Paare, so daß 43 Vermessungsregionen entstanden, fünf davon in Alaska1, wie die Karte 2 zeigt. 


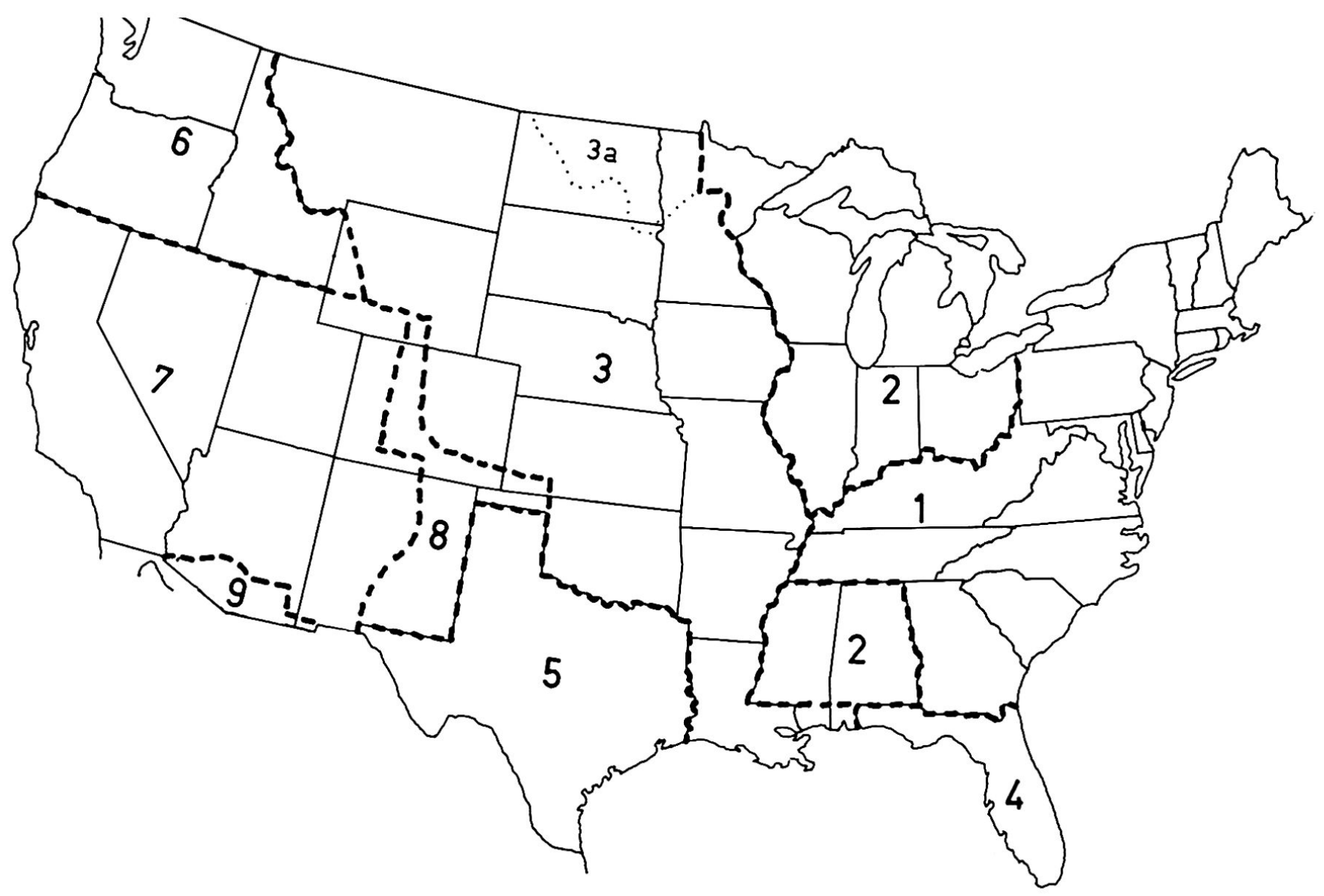

Karte 1. Das territoriale Wachstum der USA. Erklärung der Ziffern im Text

Von diesen Linien aus wurde das Land in Quadrate aufgeteilt, deren theoretische Größe sich wie folgt verstand:

1. Townships («Stadteinheiten») 36 Quadratmeilen

2. Sections

1 Quadratmeile

3. Quarter sections $=$ Homesteads

$$
\mathrm{zu} 64 \mathrm{ha}=1 / 4 \text { Quadratmeile }
$$

4. für verschiedenen Bedarf nochmals die Hälfte oder ein Viertel

Eine eingehende Beschreibung findet sich bei Boesch, USA (3, S. 65).

In den neugebildeten Townships wurde meist eine Section für Schulen reserviert, später eine zweite für die Kirchen. In diesen landesplanerischen Maßnahmen genossen die Regionen eine gewisse Selbständigkeit; so sind im Nordwesten je Township zwei Sections für die Schulen freigehalten. Schulen und Kirchen sind damit in den USA zu Großgrundbesitz gelangt.

Das quadratische Landaufteilungsmuster überzieht riesige Gebiete, beträgt doch die größte West-OstAusdehnung der Public Lands etwa $3500 \mathrm{~km}$. Bei genauerem Betrachten kann man in diesem Netz Unregelmäßigkeiten und Fehler entdecken, die drei Ursachen haben:

1. die geodätischen Korrekturen wegen der Erdkrümmung
2. die Berührungslinien zwischen den Vermessungsregionen

3. offensichtliche Vermessungsfehler, besonders im Gebirge

Bei genauerem Betrachten der Karte 3 sind einige Unstimmigkeiten dieser Art zu finden. So kommt es, daß nicht alle Landstücke die theoretische GröBe haben.

\section{Der Verkauf}

Der Verkauf geschah ursprünglich direkt durch das Schatzdepartement; 1812 erfolgte die Gründung eines eigenen Büros innerhalb des Departementes, das General Land Office; darin entlastete ein Land Commissioner den Secretary of the Treasury.

1849 wurde das Departement des Innern gegründet; es übernahm neben dem Office of Indian Affairs auch das General Land Office. Die Unterordnung der beiden Abteilungen unter den selben Staatssekretär sollte die nötige Koordination zwischen den beiden Abteilungen sichern.

1930 wurden auf Anstoß von Senator E. Taylor (Colorado) 8 Millionen Acres für Weideland aus der Public Domain abgetrennt ${ }^{8}$; mit der Zeit wurden es sogar 142 Millionen. Ein eigenes Büro, der Grazing Service, verwaltete die Weiderechte und führte 


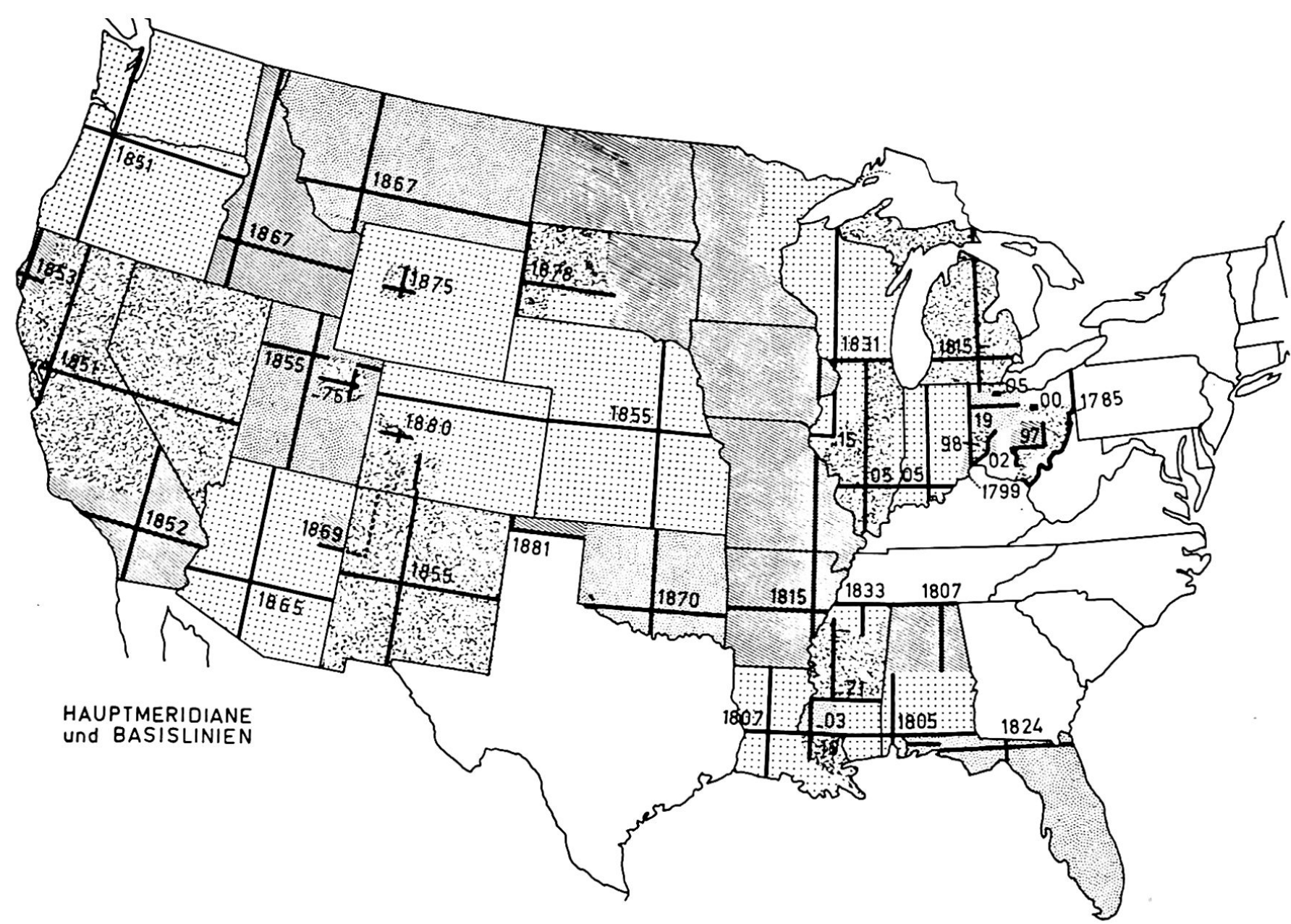

Karte 2. Die 43 Vermessungsregionen der USA mit Base Line, Hauptmeridian und Gründungsjahr ${ }^{1}$

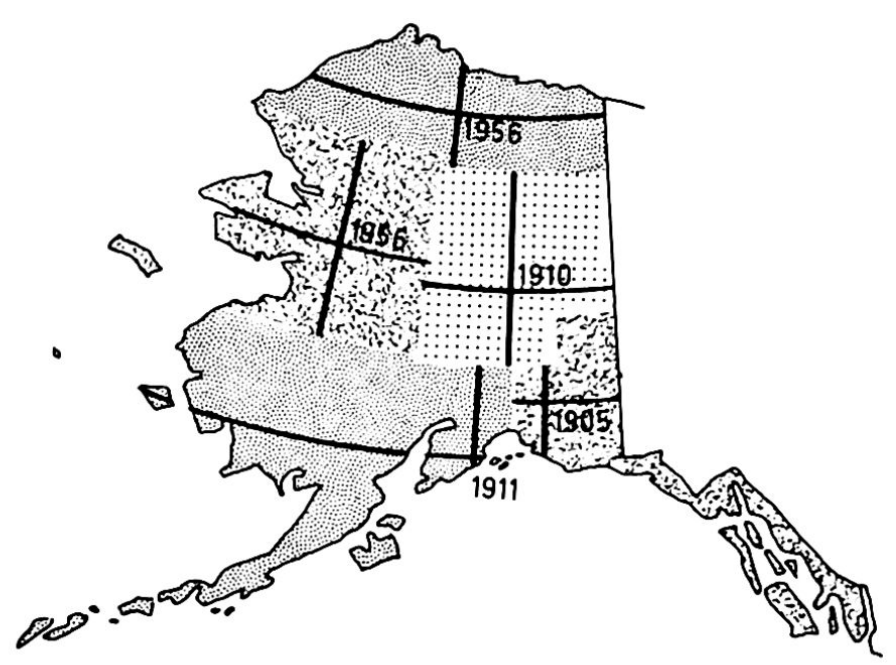

damit dem Staat einiges an Einnahmen zu; gleichzeitig konnte er vorbildliche Maßnahmen und Beweidungsvorschriften durchsetzen. Eine andere Folge waren aber Komplikationen und Doppelspurigkeiten, welche die Verkäufe behinderten. Daher wurden 1946 das General Land Office und der Grazing Service aufgehoben und durch das Bureau of Land Management ersetzt. Seine Verkaufstätigkeit ist heute minim, die übrigen Aufgaben dagegen sind in ständigem Wachsen begriffen.

\section{Die Preise}

Als das Land Office die Verkäufe aufnahm, mußte ein Siedler für 1 Acre $(0,4$ ha) Land 2 Dollar bezahlen, also 5 Dollar für die Hektare; der Einfachheit halber wurden aber nur ganze Quarter sections angeboten. Ein Siedler mußte daher über ein gewisses Eigenkapital verfügen, wenn er eine Homestead anlegen und bis zur ersten Ernte durchhalten wollte, denn die Kreditbestimmungen waren sehr hart: Dem jungen Bundesstaat fehlten die direkten Steuereinnahmen, so daß er - neben den Zöllen - auf den Erlös aus dem Landverkauf dringend angewiesen war.

Im Laufe der Zeit aber traten verschiedene Verbilligungen ein, auch wurden die Kreditbedingungen kräftig gelockert, als die direkte Einkommenssteuer eingeführt und recht ergiebig erhoben wurde. Immerhin gestattete man sich einige Ersparnisse für die Staastkasse, wobei die geübte Großzügigkeit in den ehemals spanischen Gebieten nicht angewendet wurde; so erhielt der Schweizer General Sutter in Kalifornien nach 1848 vom Land Office eine Rechnung von rund 4 Millionen Dollar, wodurch er bankrott wurde ${ }^{13}$.

1862 wurde im Homestead Law unter dem Eindruck der gewaltig gewachsenen Landreserven daß aber dieser Zuwachs jetzt praktisch zu Ende 


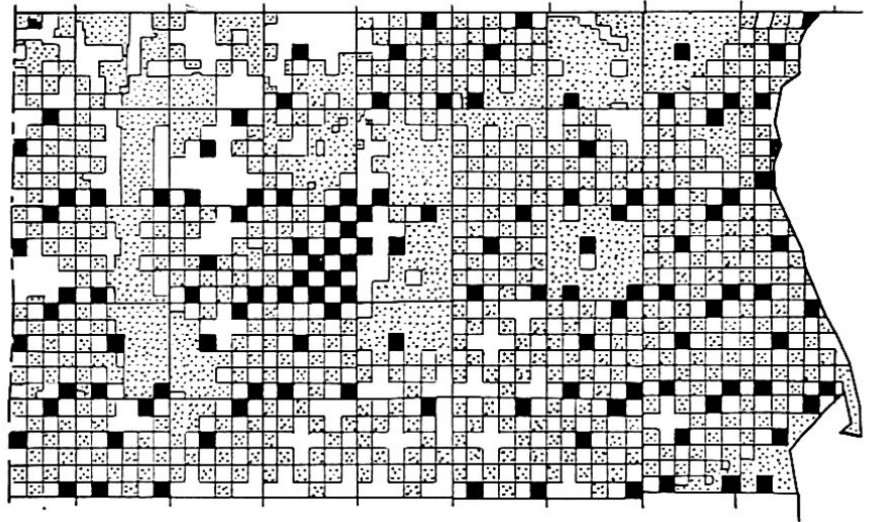

Karte 3. Landbesitzmuster in Elder County, NWUtah2. Punktiert: Public Domain (BLM); schwarz: State Land; weiß: Privatbesitz. Die Grenzen der Townships sind durchgezogen, so daß die geodätischen Korrekturen deutlich sichtbar werden. Dicke Linie rechts: Original Meander Line, d. h. die lokale Ausgangslinie für die Vermessung (NW-Rand des Großen Salzsees). In dieser Region wurden meist nur ganze Sections gehandelt. Auf Karte 4 ist dieses Gebiet weiß eingetragen

war, wurde nicht bemerkt - beschlossen, einem Siedler die Homestead von 16 Acres (64 Hektaren) gratis bzw. gegen die Bezahlung der üblichen Gebühren von 30 Dollar abzugeben. Damit verlor der Staat allerdings das Lockmittel für den Dienst in der Armee, denn während 86 Jahren waren die Veteranen durch Gratisland entschädigt worden 8 . Dieses Veteranenrecht ist übrigens erst 1959 offiziell aufgehoben worden ${ }^{11}$.

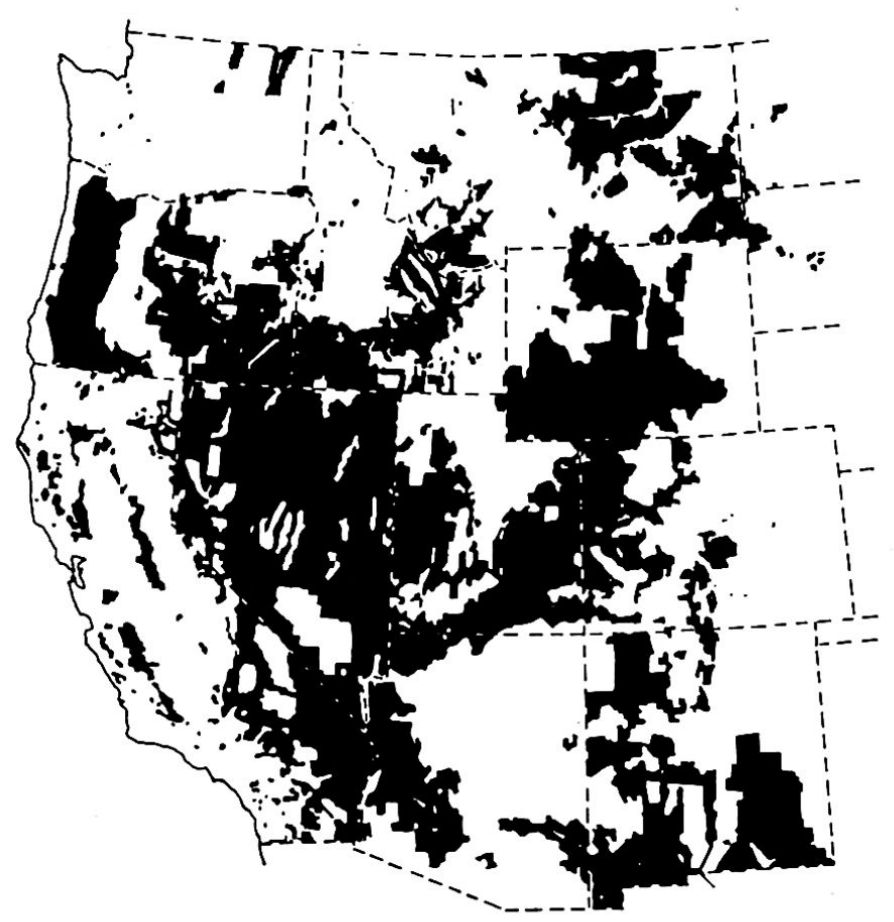

Karte 4. Der Landbesitz des Bureau of Land Management - die Public Domain - im Westen der USA ${ }^{10}$ Schwarz: Gebiete, in denen mindestens 25-50\% der Fläche dem BLM unterstellt sind
Gleichzeitig erhielten die Transkontinentalbahnen zur Verbilligung der Erschließung des Landes 91 Millionen Acres gratis, nämlich zum ersten alle Sections, die vom Bahntrassee benutzt wurden, zum zweiten zusätzliches Land - immer ganze Sections - in gestreuter Form für Landabtauschgeschäfte und Entschädigungen; daher erklärt sich der lange andauernde Reichtum der Bahngesellschaften (wenn dies heute nicht mehr der Fall ist, so darum, weil die lukrative Betätigung auf dem Immobilienmarkt juristisch vom Transportbereich abgetrennt ist).

Da höhere Schulen noch fehlten, wurden noch 11 Millionen Acres zusätzlich zum bereits reservierten Schulland bereitgestellt; darauf entstanden mit der Zeit 69 sogenannte Land Grant Colleges, die ursprünglich der Ausbildung von Landwirten und Handwerkern dienten; heute haben sie ihre Berufsschulfunktion mit Hochschulfunktionen erweitert, so daß ihre Absolventen in der Hochschulstatistik figurieren, während sie bei uns mehrheitlich zu den Berufsschülern und Berufsmittelschülern gehören würden.

Der Landzuwachs war schon ganz zum Stillstand gekommen, als der Kongreß die letzten zusätzlichen Erleichterungen schuf, die man heute sehr bereut:

1873: Wer 40 Acres Wald (10 ha) in gutem $\mathrm{Zu}$ stand hielt, durfte 160 Acres neu dazu nehmen.

1877: Wer 10 Acres Wald in gutem Zustand hielt, durfte 160 neu dazunehmen.

1877: Wer Land in Trockengebieten innert dreier Jahre bewässern wollte, erhielt 640 Acres zu 25 Cents die Acre. Zu diesen Bedingungen ist in Idaho in den letzten Jahren noch mehrmals verkauft worden.

\section{Die neuere Entwicklung}

Während das Land bedenkenlos in riesigen Mengen weggegeben wurde, formierte sich langsam ein Widerstand in Kreisen weitsichtiger Amerikaner, die schon vor hundert Jahren erkannten, daß in privaten Händen unersetzliche Werte zugrundegingen, die ein Staatswesen wohl zu bewahren gewußt hätte. Diese Neubesinnung äußerte sich 1872 in der Schaffung des ersten Nationalparkes, des YellowstoneGebietes; wegen des großen Landüberschusses erhielt er wie die meisten später gegründeten Schutzgebiete ein riesiges Areal.

1891 wurde die Waldverschacherung eingestellt mit der Schaffung der National Forests, die seit 1905 durch den US Forest Service, einer Dienststelle des Landwirtschaftsdepartementes, verwaltet werden. 1903 folgte das erste große Bundes-Wildschutzgebiet.

Um 1902 beschränkte sich der Verkauf von Staats- 
land schon auf den Westen und Alaska; durch einen Kongreßbeschluß wurden die nicht mehr reichlich fließenden Einnahmen nicht mehr der allgemeinen Bundeskasse überlassen, sondern zweckgebunden für den Bau und Unterhalt von Bewässerungsanlagen bereitgestellt. Auf dieser Grundlage konnte sich später das US Corps of Engineers entwickeln, welches die berühmten Staudämme des Landes erstellt und betreibt.

Von 1812 bis 1930 hat das General Land Office über eine Milliarde Acres (gegen 4 Millionen km²) Land an Siedler verkauft. Weitere 800 Millionen Acres wurden für National Parks, National Forests, National Wildlife Refuges und Military Reservations abgegeben. Dennoch besteht heute noch ein Fünftel des Staatsgebietes, nämlich 450 Millionen Acres, aus Public Domain8. Davon werden jährlich etwa 60000 Acres abgegeben, denen ein bescheidener Zuwachs aus verschiedenen lokalen Gründen gegenübersteht ${ }^{10}$.

\section{Der heutige Stand}

Wer heute Land aus der Public Domain kaufen will, findet kein großes Angebot mehr: In 24 Staaten gibt es kein Public Land mehr, in weiteren acht nur sehr wenig, so daß Verkäufe kaum mehr vorkommen. In den verbleibenden 11 Staaten dagegen gibt das BLM (Bureau of Land Management) periodisch bekannt, was - ohne Schaden für die Erreichung seiner Ziele - verkauft werden soll. Auf Anfrage ist eine genaue Grundstückbeschreibung erhältlich; als Ausschreibungspreis gilt ein geschätzter Verkehrswert. Findet sich ein Interessent, so wird eine öffentliche Steigerung ausgeschrieben, die in einem der 14 Land Offices stattfindet. Der Zuschlag erfolgt nur auf oder über dem Verkehrswert, den das Amt ermittelt hat, und nur gegen Bar- bzw. Checkzahlung. Im allgemeinen besteht ein beschränktes Vorkaufsrecht für die Grundstücknachbarn, d. h. wenn sie den ersteigerten Preis erlegen wollen, so erhalten sie das Land zugeschlagen.

Das zum Verkauf gelangende Areal hat nur gelegentlich "some agricultural potential»9, meist ist es Weideland oder Wald, oft Halbwüste oder alpine Matte. Zudem wird kein Zugang garantiert, dieser muß also noch mit den Grundstücknachbarn ausgehandelt werden.

Einzelstaaten, Counties und Incorporated Towns, selten auch andere Institutionen, können Land für Erholungszwecke ohne Versteigerung erwerben; der Preis wird in diesem Falle gemeinsam ausgehandelt. Vom Verkauf ausgeschlossen ist Land, das im Verdacht steht, Bodenschätze zu beherbergen.

Landverpachtung ist im Gegensatz zum Verkauf häufig, einerseits für den Weidegang, andererseits zur Ausübung von Schürfrechten samt Erstellung

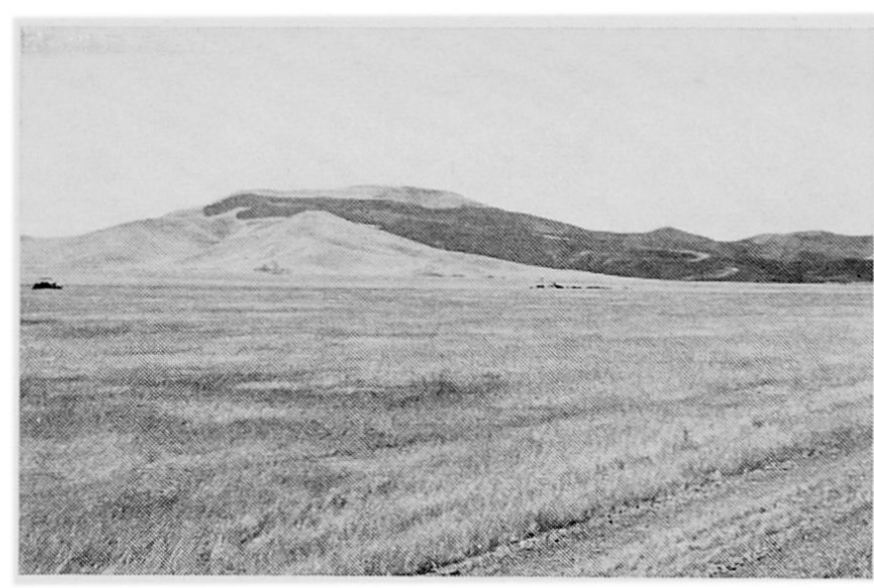

Abbildung 1. Los Banos, California (August 1963). Kurzgrassteppe am Ostrand des Küstengebirges, praktisch schadenfrei bewirtschaftet; im Mittelgrund zwei Wasserstellen im Bau. Im Hintergrund Brandfläche; die häufigen (von den Indianern früher jährlich gelegten) Brände dürften maßgeblich an der Entstehung der riesigen Prärieflächen beteiligt gewesen $\operatorname{sein}^{14}$

der nötigen Betriebsanlagen sowie für alle Versorgungsanlagen im weitesten Sinn (Straßen, Tankstellen, Motels usw.). Doch tritt der Pachterlös neben den andern finanziellen Erträgnissen der Public Domain zurück.

\section{Der heutige öffentliche Landbesitz in den USA}

Trotz des forcierten Landverkaufes sind besonders im Westen der USA noch gewaltige Gebiete in öffentlichem Besitz, so daß zum Teil landesplanerische Möglichkeiten bestehen, von denen wir uns in der Alten Welt überhaupt kein Bild machen können. Während aber zum Beispiel in der Schweiz als öffentliche Grundbesitzer die Gemeinden an vorderster Stelle stehen, ist es in den USA aus den genannten historischen Gründen der Bund. Sein riesiger Landbesitz ist aber aufgeteilt unter verschiedene Administrationszweige. Die Einzelstaaten haben sich später mit mehr oder weniger Aufwand und Erfolg ebenfalls etwas Land zugelegt, oft auch Waldgebiete. Das General Land Office ist aber nur teilweise der Lieferant, oft wurden vielmehr Areale von Privaten erworben. Über den Umfang dieses Landbesitzes sind aber kaum Angaben aufzutreiben, noch weniger über jenen der Counties und Gemeinden.

Die folgende Liste gibt die wichtigen Besitzer nicht überbauten Landes der öffentlichen Hand an 2, 6 .

\section{A. Federal Land}

1. Public Domain (Dept. of the Interior, Bureau of Land Management) 450 Millionen Acres.

2. National Parks, Monuments etc. (Dept. of the Interior, National Park Service). 
National Forests (Dept. of Agriculture, Forest Service)

National Grasslands (Dept. of Agriculture)

National Wildlife Refuges (Dept. of Agriculture, Bureau of Sport Fisheries and Wildlife)

Military Reservations (Dept. of Defence) 800 Millionen Acres

3. Indian Reservations (Dept. of the Interior, Bureau of Indian Affairs): Landtitel zum Teil in der Hand des Bundes

B. State Land

\section{State Land 2. State Forests 3. State Parks}

\section{Andere Körperschaften}

1. County Parks usw.

2. Schulkreise

3. Kirchgemeinden

Damit hat die amerikanische Öffentlichkeit ein Potential in der Hand, dessen Bedeutung sie eigentlich heute noch nicht richtig erkannt hat.

\section{Der Bedeutungswandel der Public Domain}

Oben ist darauf hingewiesen worden, daß der Staal nie alle Public Lands auf einmal besaß, sondern als Händler aufnahm und verkaufte. Der Landzuwachs erfolgte nach Westen und Nordwesten, der Verkauf drang von Osten her nach. Der augenblickliche Landbesitz des Bundes verschob sich also nach Westen und auch in andere Klima- und Vegetations- zonen; daher nahm die Qualität der Public Domain langsam ab, bis das «homesteading» praktisch unmöglich wurde. Der Bund blieb buchstäblich auf seiner Ware sitzen, er wurde vom Landhändler zum Landbesitzer. So ist auch die Zeit für das General Land Office vorbei. 1946 wird das Bureau of Land Management (BLM) gegründet, welches das General Land Office und den Grazing Service ersetzt. Dieses muß nun ganz andere, bisher für den Staat völlig ungewohnte Aufgaben erfüllen; es soll wirtschaften, aus dem Land etwas herausholen. Der wohl größte Landwirtschaftsbetrieb (im weitesten Sinne) der Welt ist entstanden, geführt von einer "management agency» 6 , in welcher das technische Personal die Beamten und Politiker ersetzt.

\section{Das Bureau of Land Management}

Erst achtzehn Jahre nach der Gründlung, 1964, war die gesetzliche Grundlage für den Großbetrieb geschaffen. Danach erfolgt die organisatorische Aufgliederung in vierzehn Regionen:

$\begin{array}{ll}\text { Südalaska } & \text { Montana, Nord- und Süddakota } \\ \text { Nordalaska } & \text { Nevada } \\ \text { Arizona } & \text { New Mexico, Oklahoma, Texas } \\ \text { Südkalifornien } & \text { Oregon, Washington } \\ \text { Nordkalifornien } & \text { Utah } \\ \text { Colorado } & \text { Wyoming, Kansas, Nebraska } \\ \text { Idaho } & \text { Osten }\end{array}$

Der größte Teil des zu verwaltenden Landes liegt in den folgenden elf Staaten ${ }^{10}$ (Karte 4):

\begin{abstract}
Alaska 278 Millionen Acres $=1,13$ Millionen $\mathrm{km}^{2}=71 \%$ der Staatsfläche
Nevada $\quad 48$ Millionen Acres $=0,19$ Millionen $\mathrm{km}^{2}=67 \%$ der Staatsfläche

Utah 23 Millionen Acres $=0,09$ Millionen $\mathrm{km}^{2}=43 \%$ der Staatsfläche

Wyoming 17 Millionen Acres $=0,07$ Millionen $\mathrm{km}^{2}=27 \%$ der Staatsfläche

Oregon 16 Millionen Acres $=0,06$ Millionen $\mathrm{km}^{2}=25 \%$ der Staatsfläche

California 15 Millionen Acres $=0,06$ Millionen $\mathrm{km}^{2}=15 \%$ der Staatsfläche

New Mexico 13 Millionen Acres $=0,05$ Millionen $\mathrm{km}^{2}=17 \%$ der Staatsfläche

Arizona 13 Millionen Acres $=0,05$ Millionen $\mathrm{km}^{2}=18 \%$ der Staatsfläche

Idaho $\quad 12$ Millionen Acres $=0,05$ Millionen $\mathrm{km}^{2}=23 \%$ der Staatsfläche

Colorado 8 Millionen Acres $=0,03$ Millionen $\mathrm{km}^{2}=12 \%$ der Staatsfläche

Montana 8 Millionen Acres $=0,03$ Millionen $\mathrm{km}^{2}=9 \%$ der Staatsfläche

Total am 30. Juni 1967 452 $1 \frac{2}{2}$ Millionen Acres, davon zwei Drittel in Alaska.
\end{abstract}

Die unterschiedlichen Anteile der Public Domain am Staatsareal erklären sich meist durch die klimatischen und vegetationsgeographischen Verhältnisse. Eine Karte der Public Domain zeigt damit annähernd die Grenzzone der Ökumene in Nordamerika an. Daneben aber treten politische Unterschiede leicht zutage. So gelang es in Oregon, im Küstengebirge riesige Wälder zurückzuhalten, während sie jenseits der Grenzen in Washington und Kalifor- nien rasch (besonders zwischen 1873 und 1895) private Besitzer fanden oder als Rettung vor dem Verkauf vom US Forest Service gesichert wurden - die beiden Departemente des Innern und der Landwirtschaft waren sich über den einzuschlagenden Weg noch nicht einig.

Die Festlegung der Aufgaben und Pflichten zeigt den außerordentlich weit gespannten Rahmen des Unternehmens ${ }^{10}$ : 
1. Wald- und Weidebewirtschaftung

2. Grundwasserschutz und -anreicherung

3. Landverkauf und -verpachtung

4. Führung der Grundbücher, soweit noch keine lokalen Behörden dafür zuständig sind

5. Ausgabe von Schürfrechten (auch auf den Schelfen)

6. Öffentliche Landvermessung

7. Schutz und Verbesserung der natürlichen Biotope für das Wild

8. Entwicklung aller Einrichtungen, die einem weitern Publikum dienen (bes. Erholungsgebiete)

9. Landschaftsschutz (Bewahrung und Wiederherstellung)

(Reihenfolge nach dem amerikanischen Original)

Die Verschiedenartigkeit der Aufgaben, im Gesetz als «multiple use principle» umschrieben, kommt so nicht einmal vollständig zum Ausdruck; denn es gehören noch Aufgaben dazu, die von vielen Einzelstaaten vernachlässigt werden, so der Gewässerschutz, Fischzucht (besonders zur Bestockung bundeseigener Stauseen), industrielle Entwicklung (bes. Holzverarbeitung, Bergbauanlagen, Kieswerke und Steinbrüche usw.), Bodenerosionsschutz und Beseitigung von Mißwirtschaftsschäden, Erschließung durch Straßen.

Die Rechnung des BLM ist in den letzten Jahren gewaltig angeschwolllen und zu einem erfreulichen Aktivposten der Staatsrechnung geworden. In den elf Jahren von 1958 bis 1968 betrugen die Einnahmen 4346 Millionen Dollar, der Aufwand nur 640 Millionen Dollar. Das letzte dieser Jahre schlug alle Rekorde mit 1159 Millionen Dollar Einnahmen und 89 Millionen Dollar Ausgaben! Den Löwenanteil bringen dabei die Schürfkonzessionen ein, und unter diesen wiederum die Ölrechte der Schelfmeere und Alaskas. Die Public Domain ist so richtig zur Milchkuh für den Staatshaushalt geworden, wobei aber mit dem rein wirtschaftlichen Nutzen erst ein Teil des Gesamtnutzens für das Staatswesen erfaßt ist.

Der relativ geringe Aufwand für die Public Domain umfaßt auch alle (voll abgeschriebenen) Investitionen und ist recht dürftig, vor allem wenn man noch berücksichtigt, daß der Ertrag sich in den letzten elf Jahrzehnten verzehnfachte, derAufwand aber nur verdreifachte. Heute bestehen deshalb fühlbare Gegensätze zwischen dem technischen Management des BLM und der Administration des Präsidenten Nixon. Ein Beispiel soll dies verdeutlichen:

Die Entwicklung der riesigen Ländereien braucht Arbeitskräfte. Zur Zeit der Administration Kennedy und Johnson wurden nun in sogenannten Job Conservation Centers arbeitslose Jugendliche aus den Städten zusammengenommen, um besonders in Waldgebieten Arbeiten in öffentlichem Intresse auszuführen. Zeltplätze mit Nebenanlagen, Wan- derwege, Waldwege und Wasserstellen wurden angelegt. Die jungen Leute kamen dadurch zu einer Art Lehre als Waldarbeiter und fanden meist nachher entsprechende Arbeit. Durch eine sinnvolle Gestaltung der Freizeit wurde aber auch versucht, gegen mitgebrachte Milieuschäden zu wirken. Das Programm war der sozialen Lage in den USA gut angepaßt und ein kleiner Beitrag zur Lösung des Slumproblems amerikanischer Städte und der Jugendarbeitslosigkeit; dennoch ist es den Sparmaßnahmen der Administration Nixon zum Opfer gefallen, da es den Reingewinn des BLM etwas schmälerte (nach Angaben eines Leiters für Weiterbildungskurse des BLM-Personals in Denver und Berichten aus Tillamook, Oregon).

\section{Die Weideverbesserung}

Unter den vielen Aufgaben, die das BLM zu erfüllen hat, sei ein relativ investitionsreiches Unternehmen herausgegriffen und besprochen, das auf lange Sicht sehr wertvoll, im Moment aber noch gar nicht einträglich ist und deswegen nicht mit der gewünschten Energie vorwärtsgetrieben werden kann: die Weideverbesserung.

Als die Europäer nach dem Westen vordrangen, fanden sie westlich von etwa $100^{\circ}$ westlicher Länge riesige Gebiete vor, die mit wogendem Gras überzogen waren, das sich in der Trockenzeit leuchtend gelb verfärbte: die Prärien.

Diese Steppen sind nur bedingt auf klimatische Ursachen zurückzuführen. Sie werden von ganz wenigen perennierenden Grasarten aufgebaut, die auf ebenem Grund einen so dichten Überzug und Wurzelfilz bilden, daß sie das Aufkommen von allen andern Pflanzen, auch von Bäumen und Sträuchern, verhindern (Abb. 1). Sobald aber die Geländeneigung leichte Bodenbewegungen erlaubt, reißt die Grasdecke auf, so daß sich sofort trockenresistente Bäume ansiedeln, so etwa nördlich $40^{\circ}$ Breite in den ganzen Prärien Pinus- und Juniperus-Arten (Vortrag von Dr. P. Peisl 1969, Geobot. Inst. Rübel der ETH, Zürich).

Diese perennierenden Gräser zeigen eine starke Empfindlichkeit auf Beweidung; in einem Jahr dürfen höchstens 50\% einer Pflanze abgefressen bzw. abgetreten werden, sonst stirbt sie unweigerlich ab, da sie sich in der kurzen Vegetationsperiode nicht erholen kann 7 . Die entstehenden Lücken werden sofort durch Buschvegetation aufgefüllt, in welcher Wermut (Artemisia tridentata) dominiert, während sich in den Zwischenräumen annuelle Gräser und Kräuter ansiedeln. Die letzten wandern bei bloßliegendem Boden sehr rasch ein; oft sind es aus dem europäischen Mittelmeergebiet eingeschleppte Gräser, die mit nur drei bis sechs Tagen Keimzeit die einheimischen Arten zu verdrängen mögen 12; 


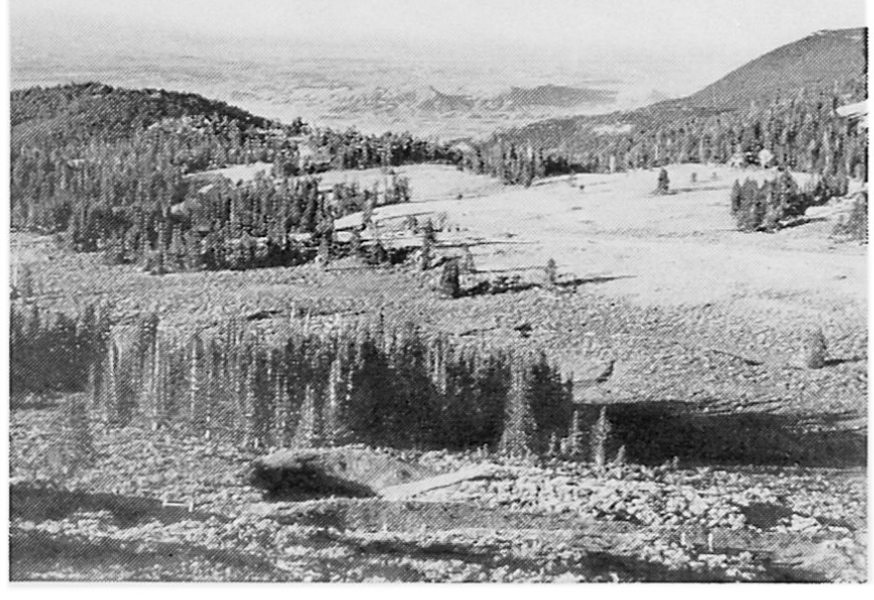

Abbildung 2. Bighorn-Gebirge, E Lovell, Wyoming (Aufnahme Dr. P. Peisl, Juli 1969). An feuchtern Stellen Nadelholzbestände, sonst Steppe des nördlichen Prärietyps 14. Am Farbwechsel bzw. Dunklerwerden ist das Vordringen von Artemisia in die geschädigte Rasendecke einer Schafweide gut zu erkennen

da sie aber nach der Vegetationsperiode samt Wurzeln rasch zerfallen, haben sie keinen Wert als Futterpflanze oder Bodenfestiger. Der Vorgang des Vegetationswechsels ist rasch: Schrumpft einmal der Futtergrasbestand einer Weide, so geht das Vieh nicht an die neuen Pflanzen, sondern meidet sie wegen ihres Geschmacks (außer dem Wermut breitet sich so im Südwesten auch der Kreosottbusch [Larrea tridentata]* und der «rabbit brush» [Chrysotamnus nauseosus] kräftig aus), schädigt also die verbleibenen perennierenden Gräser erst recht; die "natürliche Auslese» ist daher sehr wirkungsvoll (Abb. 2). Die neue Pflanzengesellschaft ist aber ein schlechter Wasserabsorber, was die Grundwasseranreicherung schwer beeinträchtigt und durch ihre Pfahlwurzeln ein schlechter bis untauglicher Bodenerosionsschutz, so da $\beta$ heftige Gewitter zu schichtflutähnlichen Erscheinungen führen. Die Bodenerosion aber erschwert wiederum eine Rückführung in den ursprünglichen Zustand.

Das BLM versucht nun die Sanierung degenerierter Weidegebiete. Zuerst muß der Kleinstrauchbestand vernichtet werden. Je nach Klima (Stärke der Regenfälle) und Bodenneigung wird der Wermut (zusammen mit allen andern Dikotyledonen) durch Unkrautvertilger abgetötet, oder er wird mit Spezialpflügen nach unten gewendet (Abb. 3). Sind noch genügend perennierende Gräser da, so setzen sie sich im ersten Fall bei Weideverbot rasch durch und nehmen bald wieder das ganze Areal ein; im andern Fall muß frisch angesät werden. Meistens kann nach zwei bis drei Vegetationsperioden mit durchschnittlichen Niederschlägen wieder die erste Beweidung folgen. Wenn die Weiderechte nun vernünftig gehandhabt werden, kann in großen Gebieten der Bodenerosion wieder Einhalt geboten werden, der Grundwasserstand regeneriert sich, und auch der Wildbestand nimmt sprunghaft zu, dies alles trotz gleichzeitiger Steigerung der Viehproduktion. Für den Amerikaner im Westen aber steht damit die Erkenntnis fest, daß nicht nur Waldland, sondern auch Weideland, d. h. die Steppengebiete, dann am besten bewirtschaftet werden, wenn die öffentliche Hand, in diesem Falle der Bund, für eine optimale Nutzung sorgt. Die Mehrzahl der Bürger läßt sich heute diesen Staatseingriff gefallen, der so sehr den Grundsätzen des amerikanischen Lebens widerspricht, da sie nicht mehr in der Urproduktion beschäftigt ist und daher im staatlichen Landbesitz keine Beeinträchtigung eigener Grundansprüche empfindet. Vielmehr fühlt sich ein Stadtbewohner fast ein bißchen als Mitbesitzer und Nutznießer, denn auf diesem Land und in dessen Gewässern leben Wild und Fische, denen er im Rahmen der lokalen Bestimmungen nachstellen darf.

Natürlich sind die Probleme der Weidegebiete nicht gelöst, solange nur das Staatsland gut bewirtschaftet ist. Den Maßnahmen kommt nun aber eine Eigentümlichkeit der Landverkäufe durch das frühere General Land Office zustatten: Über weite Strecken wurde im Westen nur jede zweite Homestead verkauft, so daß ein schachbrettförmiges Landbesitzmuster entstand (Karte 3). Dadurch haben die Ranchbesitzer heute die Möglichkeit, mit ihrem Vieh auf BLM-Land auszuweichen, während sie die Sanierung ihres eigenen Landes versuchen. Gezielte Tausch- und Rückkaufaktionen in den Steppengebieten der Gebirge vergrößern die Erfolgswahrscheinlichkeit noch zusehends.

Das erwähnte Landbesitzmuster macht hier noch eine Bemerkung zur Karte 4 nötig: Sobald ein Schachbrettmuster-Gebiet über 25\% BLM-Land aufweist, ist es schwarz eingetragen, was allerdings einen etwas verfälschten Eindruck hinterläßt.

\section{Die Anstrengungen des BLM in Oregon}

In diesem Staate ist ein Viertel des Areals dem BLM unterstellt, ein weiteres Viertel besteht aus National Forests; dazu kommen noch drei kleinere Nationalpärke und eine große Zahl von (kleinen) State Parks, so daß etwa $55 \%$ der Staatsfläche in öffentlicher Hand liegen. Das Büro in Portland hat in den letzten zehn Jahren schon einiges fertiggebracht 4 : So wurden $6000 \mathrm{~km}$ Erschließungsstraßen gebaut; jedes Jahr werden $60 \mathrm{~km}^{2}$ durch Bodenerosion oder sonst geschädigtes früheres Waldland 


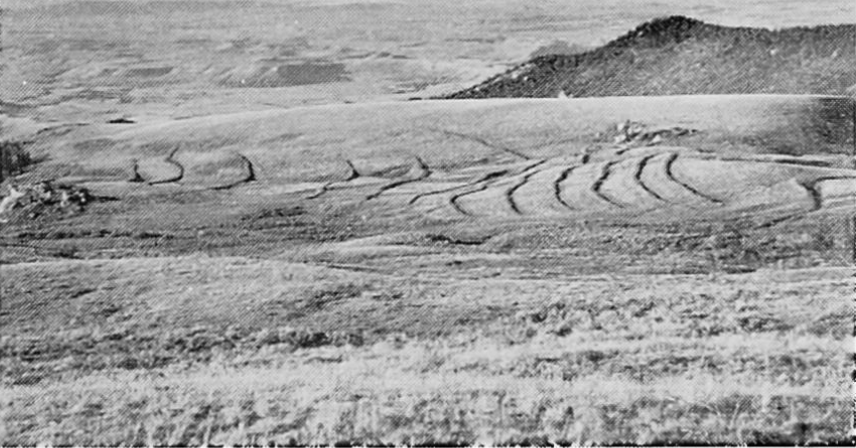

Abbildung 3. Bighorn-Gebirge, E Lovell, Wyoming (Juli 1969). Wiederhergestelltes Grasland; zur Verhinderung der Bodenerosion in der Zeit zwischen der Beseitigung der Artemisien und dem Aufwachsen der perennierenden Gräser wurden mit einem Spezialpflug waagrecht liegende Wälle aufgeworfen, die das Regenwasser zurückhalten

wieder aufgeforstet; die Steppensanierungen umfassen jährlich sogar das Doppelte; doch sind in beiden Fällen die Flächen noch viel zu gering, welche behandelt werden können. Durch verschiedene Maßnahmen (u. a. Anlage von Wasserstellen) wurden Sommerweiden für 400000 Rinder gewonnen, während im Winter hier bereits sehr viel Wild hinkommt. Die Finanzierung ist mehr als gesichert, bringen doch die Weiderechte jedes Jahr 300000 Dollar ein, der Holzverkauf gar 40 Millionen Dollar.

Mit großer Verspätung beteiligt sich das Büro nun auch am Ausbau Oregons zum großen Ferienparadies; so konnten bis 196930 meist kleine Zeltplätze und 42 Picknickplätze dem Publikum zur Verfügung gestellt werden. Wie groß der Rückstand allerdings ist, zeigen einige Vergleichszahlen: In den National Forests sind schon 550 Zeltplätze und 17 "Ski Areas» eröffnet, in den State Parks 200 und in den County Parks weitere 300 Zeltplätze, ja sogar die Kraftwerk- und Holzgesellschaften übertreffen mit 50 Plätzen das BLM bei weitem ${ }^{5}$. Zum Teil hängt dies natürlich mit dem Charakter des verwalteten Landes zusammen, doch die Verantwortlichen sind überzeugt, daß sie dazu berufen seien, den erholungssuchenden Städtern weitere große Möglichkeiten zur sinnvollen Freizeitgestaltung zu erschließen. Damit wird das BLM Werte schaffen, die in Geld überhaupt nicht ausgedrückt werden können.

\section{Literatur}

1 US Dept. of the Interior, Bureau of Land Management (1968): Principal Meridians and Base Lines Governing the Unites States Public Land Surveys (Karte).

2 US Dept. of the Interior, Bureau of Land Management, Salt Lake District (1968): Northwest Utah, Recreation and Wildlife on BLM Lands (Karte).

3 Boesch, Hans (1956): USA, die Erschließung eines Kontinentes.

4 US Dept. of the Interior, Bureau of Land Management (1967): BLM Recreation Guide, Oregon (Karte).

5 Oregon State Highway Department, Travel Information Division (1969): Oregon Parks (Karte).

6 US Dept. of the Interior, Bureau of Land Management (1968): Staking a Mineral Claim on Federal Lands.

7 US Dept. of the Interior, Bureau of Land Management (1962): Sagebrush and Annual Grasses to Perennial Grasses.

8 US Dept. of the Interior, Bureau of Land Management (1966): The Public Domain.

9 US Dept. of the Interior, Bureau of Land Management (1968): How to Buy Public Lands. Information Bull., No. 4.

10 US Dept. of the Interior, Bureau of Land Management (1968): What Are the Public Lands? Information Bull., No. 1.

11 US Dept. of the Interior, Bureau of Land Management, Oregon State Office (1969): You asked about Government Land in Oregon and Washington (Informationsblatt, hektogr.).

12 Burcham, L. A. (1970): Ecological Significance of Alien Plants in California Grass Lands. Proc. Ass. Am. Geogr. 1970/2.

13 Stucki Laurenz (1969): Das heimliche Imperium. Bern.

14 Knapp Rüdinger (1965): Die Vegetation von Nord- und Mittelamerika. Stuttgart. 